\title{
AN ENERGY EFFICIENT LEVEL BASED Clustering Routing Protocol for Wireless SENSOR NETWORKS
}

\author{
Meenakshi Diwakar ${ }^{1}$ and Sushil Kumar ${ }^{2}$ \\ School of Computer and System Sciences \\ Jawaharlal Nehru University, New Delhi, India \\ ${ }^{1}$ meenakshi.d02@gmail.com, ${ }^{2}$ skdohare@yahoo.com
}

\begin{abstract}
Nowadays advanced technology of Wireless Sensor Networks used in many applications like health, environment, battle field etc. The sensor nodes equipped with limited power sources. Therefore, efficiently utilizing sensor nodes energy can maintain a prolonged network lifetime. One of the major issues in sensor networks is developing an energy-efficient routing protocol to improve the lifetime of the networks. In this paper, we propose EELBCRP (Energy-Efficient Level Based Clustering Routing Protocol), a protocol for wireless sensor networks. Network partitioned into annular rings by using various power levels at base station and each ring having various sensor nodes. Also consider the residual energy of each node and distance from the BS of nodes as the principle of cluster-head election. The mathematical formulae for election the cluster head is provided. The model developed is simulated in MATLAB. The results are obtained in terms of three metrics- lifetime of the network, number of clusters and energy consumption of clusters heads. From the results of simulation, it is observed that the performance of EELBCRP is better in terms of energy consumption of $\mathrm{CH}$, number of clusters and lifetime of network compared with LEACH.
\end{abstract}

\section{KEYWORDS}

Wireless Sensor Networks, Energy Efficiency, Network Lifetime, clustering, LEACH Protocol,

\section{INTRODUCTION}

Wireless Sensor networks (WSN) is a large network which is consist of huge number of sensor nodes and these nodes are directly interacting with their environment by sensing the physical parameters such as temperature, humidity, etc[1]. All the sensor nodes send or receive data to/from a fixed wired station called base station (BS). The base station usually serves as a gateway to some other network. WSNs have a comprehensive range of applications in this field including $[6,9,10]$; environmental applications, military applications, home security, etc.

The main challenge is related to the limited, usually unrenewable energy supply of the sensor nodes. Hence, the available energy at the nodes should consider as a major constraint while designing the routing protocols.

Hierarchical-based routing protocols also known as cluster based routing protocols. This type of protocols enforces a structure on the network to use the energy efficiency, extend the lifetime and scalability. In this protocol, nodes of the network are organized into the clusters in which higher energy nodes (e.g. assume the job of the cluster head) can be used to process and forwarding the 
information, while lower energy nodes can be used to do the sensing the target. Clustering is an efficient way to reduce energy consumption and extend the life time of the network, doing data aggregation and fusion in order to reduce the number of transmitted messages to the BS [2]. This paper presents an extension to the protocol EEHCRP [13] based on different power levels for Wireless Sensor Networks. The proposed protocol EELBCRP reduces the number of dead nodes and the energy consumption, to extend the network lifetime.

The rest of the paper is organized as follows.An overview of related work is given by section 2 . In section 3, propose an energy efficient level based clustering routing protocol. Simulations and results of experiments are discussed in the section 4. In section 5, concludes the work presented in this paper and the scope of further extension of this work.

\section{RELATED WORK}

The first hierarchical routing protocol for WSN is Low Energy Adaptive Clustering Hierarchy $(\mathrm{LEACH})$. LEACH is a cluster-based routing protocol which includes cluster formation in distributed manner. In LEACH [3], the nodes form themselves into local clusters, with one node acting as the local cluster-head. LEACH includes randomized rotation of the high-energy clusterhead position such that it rotates among the several sensors nodes in order to not deplete the battery of a single sensor. In addition, CHs performs local data fusion to "compress" the amount of data arriving from the nodes that belong to the respective cluster and transmit aggregate data to the base station, further reducing energy dissipation and enhancing system lifetime.

In $\mathrm{LEACH}$, the cluster head receive data directly from each node and the sink uses single-hop routing. Therefore, it is not applicable for large networks. Also, it is not obvious how the number of predetermined number of cluster heads is going to be uniformly distributed through the network. Therefore, it is possible no or lots of CHs selected and also possible that too many CHs are located in a specific area. Furthermore, the dynamic clustering routing implemented with extra overhead, e.g. cluster head changes, advertisements etc., which consumed more energy.

LEACH-C protocol is the extended version of LEACH protocol. In which, all nodes in the network transmit their information to the BS, includes their ID, remaining energy, and position information. After this, the BS calculate the average energy of the network and select a set of $\mathrm{CHs}$ that have more energy than the average energy of the network and sends information about $\mathrm{CHs}$, their members and TDMA schedule. The member nodes decide own TDMA slot and transmit data in its time slot [4].A non-sovereign cluster-head selection is the main drawback of this protocol. Moreover, LEACH-C needs location information of all nodes in the network. However, the location information in wireless sensor networks is only available through GPS (Global positioning system) or a location sensing technique, such as triangulation which requires additional communication among the nodes [5].

Power-efficient gathering in Sensor Information Systems (PEGASIS) is an enhancement of the LEACH protocol. A single node in a chain is used by PAGASIS to send data to BS rather than multiple nodes. The chain is constructed in a greedy way. Each node only communicates with their closest neighbours along the communication chain. Gathered data moves from node to node, aggregated and finally transmit to the BS [6].In PAGASIS, Each sensor node is required to have additional local information about the wireless sensor network. When the PEGASIS protocol selects the head node, there is no consideration about the energy of nodes, location of the BS. This applies to the greedy algorithm for construct chain, some delay may occur. Since the head node is a single, it may happen to a bottleneck at the head node. 
International Journal Of Advanced Smart Sensor Network Systems ( IJASSN ), Vol 2, No.2, April 2012

In [11], clustering of network is done symmetrically and cluster head node is selected by the comparisons of remaining energy and distance with the other nodes. Determine the cluster head of next hope by using the weight function in [12].

\section{EELBCRP: ENERgY EFFicient LeVEL BASEd Clustering Routing PROTOCOL}

Hierarchical clustering algorithms are very important to increasing the lifetime of network. We propose EELBCRP (Energy Efficient Level Based Clustering Routing Protocol), which is a hierarchical clustering routing protocol. EELBCRP reduces the number of dead nodes and the energy consumption to extend the network lifetime. Before studying the details of the proposed algorithm, we define the expected network model and energy model.

\subsection{Network Model}

Let us consider a sensor network, consisting of $\mathrm{n}$ sensor nodes, which are randomly deployed over in an area of wireless sensor network. To prepare the network model, the following assumptions are made about sensor nodes.

Assumptions:

1. There is one base station which is fixed and located at middle in a given sensor network.

2. All sensor nodes are fixed and homogeneous with a limited stored energy.

3. Base station can transmit various power levels.

4. The sensed data by the sensor nodes are routed to the base station.

5. Each node is equipped with power constrain capabilities and vary their transmitted power.

6. Nodes are not equipped with GPS unit.

\subsection{EELBCRP Algorithm}

In this section, we describe our protocol in detail. This protocol is divided into three phases, setup phase, cluster setup phase and inter cluster routing phase.

\subsubsection{Setup phase}

On the initial deployment, the base station (BS) transmits a level-1 signal with minimum power level. All nodes, which hear this message, set their level as 1. After that, the base station increases its signal power to attain the next level and transmit a level-2 signal. All the nodes that receive the massage but do not set the previous level set their level as 2 .

This procedure continuous until the base station transmits corresponding massages to all levels. The total number of messages of levels is equivalent to the number of distinct transmit signal at which the BS can sends [7].

BS broadcast a hello massage, fig [1]. This massage contains the information of upper limit and lower limit of each level.

\begin{tabular}{|l|l|l|l|l|}
\hline $\mathrm{Ui}, \mathrm{Li}$ & $\ldots \ldots \ldots \ldots \ldots . . . .$. & U3, L3 & U2, L2 & U1, L1 \\
\hline
\end{tabular}

Figure 1.Structure of hello message 
Where

Ui: Upper limits of level i

Li: Lower limit of level i

Each node calculates the distance from the BS based on the received signal strength.

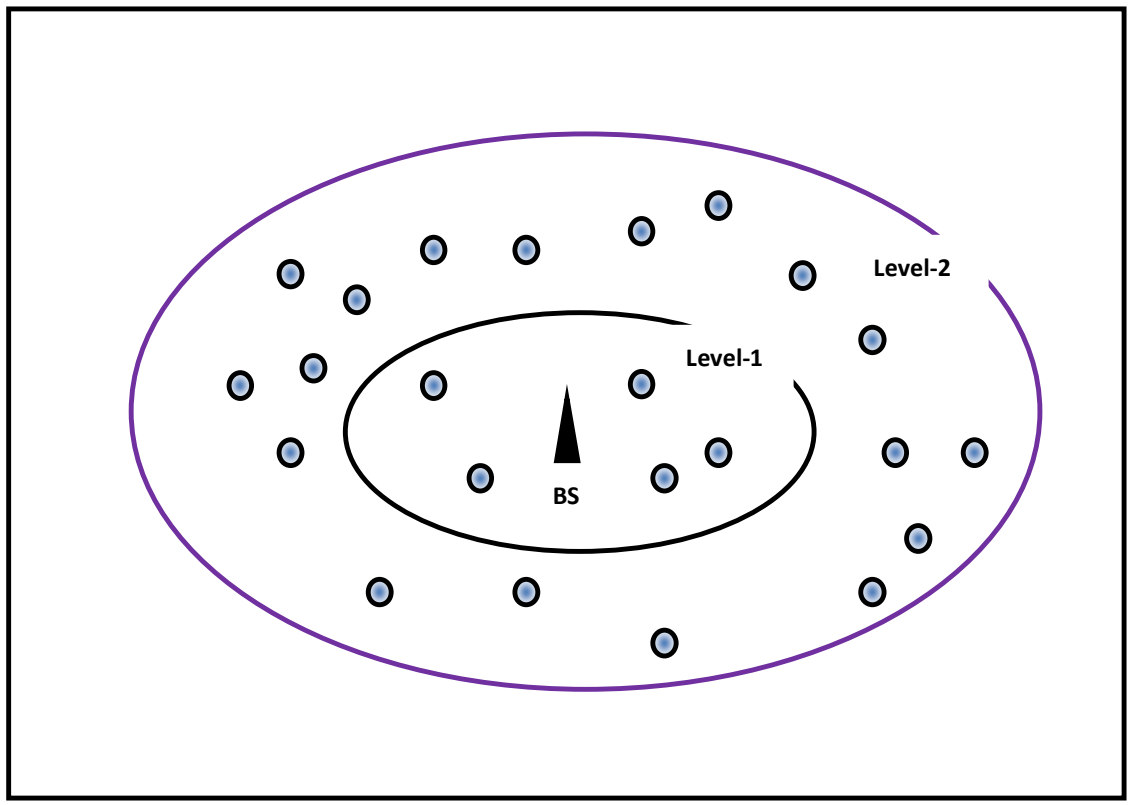

Algorithm 1. Setup phase

\#No. of nodes $\mathrm{N}$

\# BS can transmit i levels; $\mathrm{i} \geq 1$

1. For each level i, message transmitted by BS

2. If (Nodes does not assign previous level and hear new message or BS transmit level $\mathrm{i}=1$ )

3. Assign level i

4. End if

5. End for

6. BS broadcast hello message, which contains the information of upper limit and lower limit of each level.

7. Each node calculates the distance from the BS based on received signal strength

\subsubsection{Cluster setup phase}

In this phase, each level is divided into clusters. The operation of cluster-setup phase is the same as LEACH [3] except the difference of threshold formula. For each level i, each node decide whether or not to become a cluster head for the current round by choosing a random number $\mathrm{x}$ between 0 and 1.The node becomes a cluster head for the current round if this number is less than the threshold $\mathrm{T}_{\mathrm{i}}(\mathrm{n})$. The threshold defined as. 


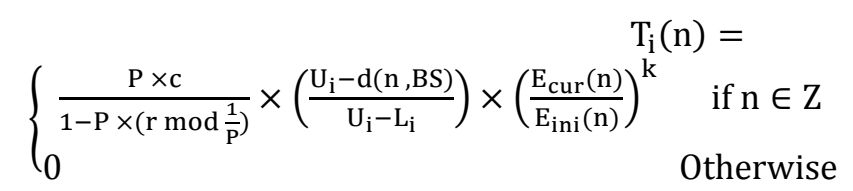

Where

$\mathrm{P}=$ the desired percentage of the cluster heads.

$\mathrm{r}=$ the current round.

$\mathrm{Z}=$ the set of nodes which have not been $\mathrm{CHs}$ in the last 1/P rounds.

$\mathrm{c}=$ the constant factor between the 0 and 1 .

$\mathrm{Ui}=$ the upper limit of level-i.

$\mathrm{Li}=$ the lower limit of level-i.

$\mathrm{d}(\mathrm{n}, \mathrm{BS})=$ the distance between node $\mathrm{n}$ and base station.

$\left.E_{\text {cur }}(n) n\right)=$ current energy of node $n$.

$E_{\text {ini }}(n)=$ initial energy of node $n$.

$\mathrm{k}=0,1,2,3$

Each node that elected itself a cluster head for the current round, broadcast an advertisement message to the rest of the node by using CSMA Mac protocol. All cluster heads broadcast their advertisement message with the same transmit energy. All non- cluster head nodes receiving these messages from all cluster head nodes and each non-cluster node decided the cluster to which it will belong for the current round. This decision is based on received signal strength of the advertisement messages. Each node must inform to the cluster head that it will be a cluster member by using CSMA Mac protocol. After that, each cluster head creates a TDMA schedule for its cluster members. This information is broadcasted back to the nodes in the cluster. Once the clusters are created and TDMA schedule is fixed, data transmission can begin. Each cluster member can be turned off until the node's allocated time.

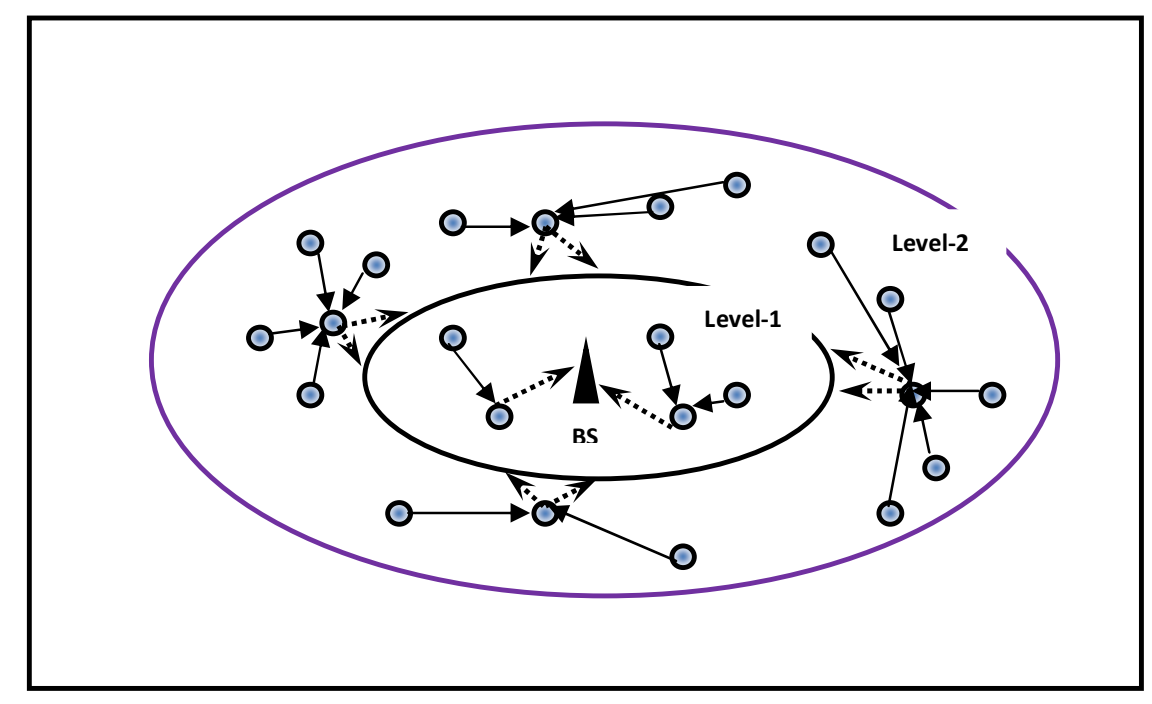

Figure 3.Cluster formation

Each node sends data to its cluster heads with minimal transmission power. This power is estimated by received signal strength of the advertisement message. So that data transmission uses a minimal amount of energy. 
When all the data has been received from the cluster members, then cluster head node perform data aggregation function to compress the data into a single signal. After a certain time the next round begin.

\section{Algorithm 2. Cluster setup phase}

1. for each (node $\mathrm{N}$ )

2. $\mathrm{N}$ selects random number $\mathrm{x}$ between 0 and 1 .

3. If $(\mathrm{x}<\mathrm{T}(\mathrm{n}))$

4. $\mathrm{N}$ becomes $\mathrm{CH}$.

5. $\mathrm{N}$ broadcasts an advertising message for its $\mathrm{CH}$ status.

6. Else

7. $\mathrm{N}$ becomes a $\mathrm{NCH}$ node.

8. $\mathrm{N}$ chooses the $\mathrm{CH}$, this selection is based on the received signal strength of advertise.

9. $\mathrm{N}$ informs the selected $\mathrm{CH}$ and become a member of its cluster.

10. End if.

11. for each $(\mathrm{CH})$

12. $\mathrm{CH}$ creates TDMA schedule for each cluster member.

13. Each cluster member communicates to the $\mathrm{CH}$ in its time slot.

14. End for

\subsubsection{Inter cluster routing}

After the cluster formation, the cluster heads broadcast the aggregate data to the next level. At the next level, the nodes aggregate their data and sends to their cluster heads.

In this manner the cluster heads at the last level transmit the final information to the BS.

\section{Algorithm 3.Inter cluster routing}

1. For each (level i)

2. for each $\mathrm{CH}$

3. $\mathrm{CH}$ receives the data from the cluster member

4. Aggregate the data.

5. If ( $\mathrm{i}==1$ )

6. $\mathrm{CH}$ transmits data to the BS.

7. Else

8. CH broadcasts data in the next level.

9. End if

10.End for

11.End for

\subsection{Energy Model}

We use a free space model. This model is used to calculate the power of received signal of each packet. There is only one clear line of sight path between receiver and transmitter is assumed by the free space propagation.

The energy consumed during the transmission is the main part of the total energy consumption. The received signal power in free space at a distance $r$ is calculated by using the following equation [8]. 
$p_{r} d B m=p_{t} d B m+10 \log _{10}\left(G_{l}\right)+20 \log _{10}(\lambda)-20 \log _{10}(4 \pi)-20 \log _{10}(r)$

Where the transmitted signal power is denoted by $\mathrm{p}_{\mathrm{t}}$, product of receive and transmit antenna field radiation patterns in the line-of-sight (LOS) direction is $\mathrm{G}_{1}$ and $\lambda$ is the carrier wavelength.

The minimum transmission power level $\mathrm{p}_{\mathrm{t}-\mathrm{min}}$ at the sender is calculated as.

$p_{t \_m i n} d B m=p_{r_{-} \min } d B m-10 \log _{10}\left(G_{l}\right)-20 \log _{10}(\lambda)+20 \log _{10}+20 \log _{10}(r)$

from (2) and (3), we obtain.

$p_{t \_m i n} d B m=p_{r_{\_} \text {min }} d B m-p_{r} d B m+p_{t} d B m$

Where $\mathrm{p}_{\mathrm{r}-\mathrm{min}}$ is the receiver's sensitivity?

The non-cluster head nodes calculate the strength of the advertisement messages from equation (2) and join the cluster which has the maximum strength of the received signal. These nodes also calculate the minimum transmission power for sending data to the cluster head with the help of eqn (4).

In free space model, to transmit a 1 bit message over the distance $r$, transmission energy consumption $\mathrm{E}_{\mathrm{T}(\mathrm{x})}(\mathrm{l}, \mathrm{r})[3]$ is-

$\mathrm{E}_{\mathrm{T}(\mathrm{x})}(\mathrm{l}, \mathrm{r})=\mathrm{E}_{\mathrm{T}(\mathrm{x}-\mathrm{elec})}(\mathrm{r})+\mathrm{E}_{\mathrm{T}(\mathrm{x})-\mathrm{amp}}(\mathrm{l}, \mathrm{r})$

$\mathrm{E}_{\mathrm{T}(\mathrm{X})}(1, \mathrm{r})=\mathrm{E}_{\mathrm{elec}} * 1+\varepsilon_{\mathrm{amp}} * 1^{*} \mathrm{r}^{2}$

where $\mathrm{E}_{\mathrm{T}(\mathrm{x}) \text {-elec }}$ is the energy dissipated by the transmitter electronics and $\varepsilon_{\mathrm{amp}}$ is the energy dissipated by the transmit amplifier.

$\mathrm{E}_{\mathrm{R}_{\mathrm{x}}}(\mathrm{r})=\mathrm{E}_{\mathrm{R}_{\mathrm{x}-\text { elec }}}(\mathrm{r})$

$\mathrm{E}_{\mathrm{R}_{\mathrm{x}}}=\mathrm{E}_{\text {elec }} * \mathrm{r}$

where $\mathrm{E}_{\mathrm{R}_{(\mathrm{x})-\text { elec }}}$ denote the receiver electronics.

\section{SIMULATION RESULTS}

In this section, the simulated results are obtained to evaluate the performance of EELBCRP using MATLAB. We simulated the energy consumption, number of clusters and resulting lifetime of the network. Firstly we evaluated the performance of EELBCRP for different value of $\mathrm{k}$ and find the optimum value of $\mathrm{k}$. Then we compared the performance of EELBCRP with LEACH. The results obtain in terms of three metric: energy consumption of $\mathrm{CHs}$, number of clusters and life time of WSN are represented in form of graphs.

We assume that 100 sensor nodes are randomly deployed over $100 \times 100 \mathrm{~m}$ square area sensor field and the whole network is divided in three levels $(n=3)$. The BS located at $(50,50)$. The initial energy of each node is $.05 \mathrm{~J}$ and a node is considered dead when its energy is less than equal to 0 . 
International Journal Of Advanced Smart Sensor Network Systems ( IJASSN ), Vol 2, No.2, April 2012

Table 1. Shows the simulation parameter

\begin{tabular}{|c|c|}
\hline Parameters & Value \\
\hline Network size & $100 \times 100 \mathrm{~m}$ \\
\hline BS station & $(50,50)$ \\
\hline Number of sensor nodes & 100 \\
\hline Initial energy & $.05 \mathrm{~J}$ \\
\hline $\mathrm{E}_{\text {elec }}$ & $50 \mathrm{~nJ} / \mathrm{b}$ \\
\hline$\varepsilon_{\mathrm{mp}}$ & $10 \mathrm{pJ} / \mathrm{b} / \mathrm{m}^{2}$ \\
\hline EDA & $5 \mathrm{~nJ} / \mathrm{b} / \mathrm{signal}$ \\
\hline Data packet size & $4000 \mathrm{bits}$ \\
\hline n (level) & 3 \\
\hline
\end{tabular}

\subsection{Evaluation the performance of EELBCRP for different value of $k$}

Fig.[4] shows that the number of cluster for $k=2$ are fewer than the number of clusters for $k=0,1,3$ and also observed that there is no cluster in some round for $\mathrm{k}=3$. So that our protocol is better for $\mathrm{k}=2$ than the other values of $\mathrm{k}$.

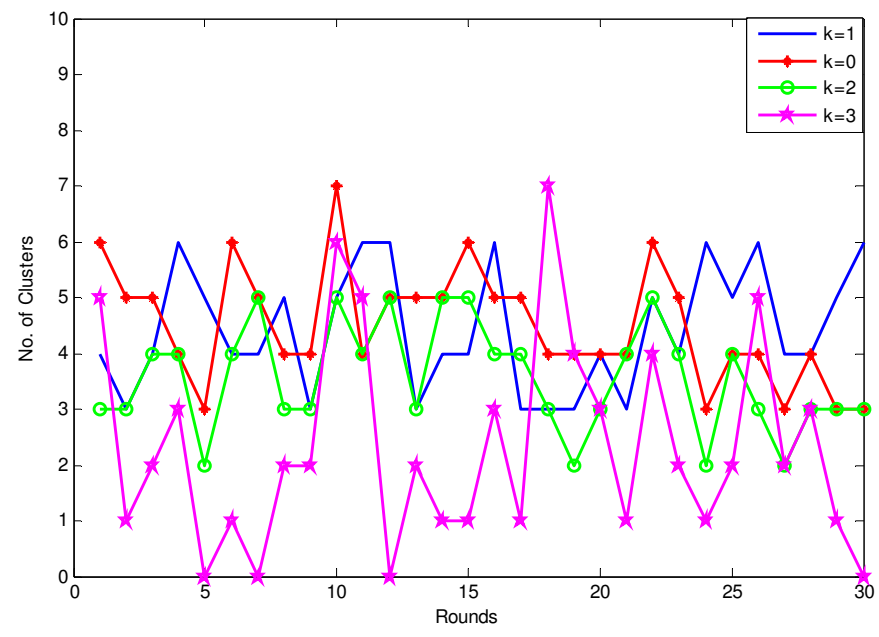

Figure 4. Optimum value of $\mathrm{k}$

Now we compared the performance of our protocol for $\mathrm{k}=2$ (say EELBCRP-2) with LEACH.

\subsection{Energy consumption of cluster heads (CHs)}

Fig.[5] shows the results for the energy consumed by CHs in EELBCRP-2 and LEACH protocol for 30 rounds. The energy consumed by $\mathrm{CHs}$ for each round in EELBCRP-2 is much lower than that in LEACH. This is due to fact that in $\mathrm{LEACH}, \mathrm{CHs}$ transmit their data direct to the BS. Therefore, the energy consumption is much higher. In EELBCRP-2, CHs sends their data to the BS through multihop communication. So a significant amount of energy is saved. For example, after the 20 rounds, the LEACH consumed the about $42 \%$ of the initial energy while in EELBCRP-2 is about $15 \%$. 


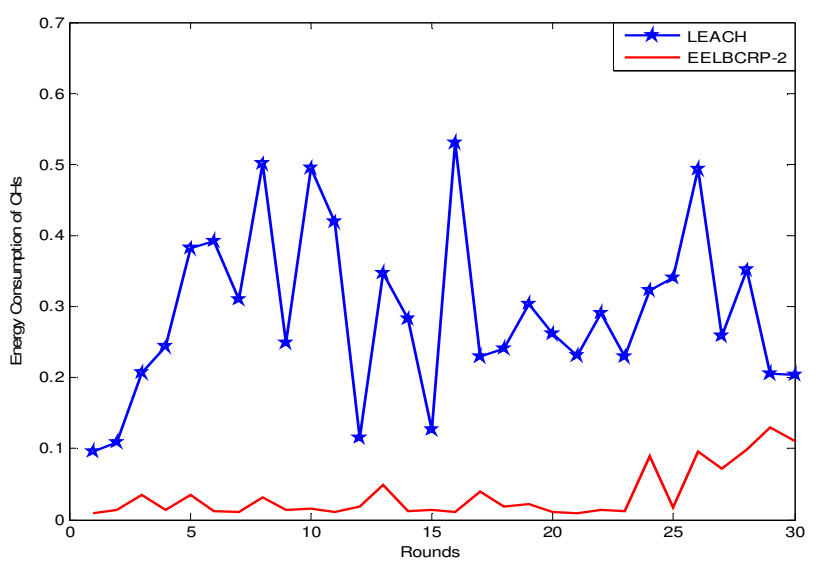

Figure 5. Energy Consumed by CHs

\subsection{Number of Clusters}

Fig [6] shows the distribution of the number of clusters in EELBCRP-2 and LEACH for 30 rounds. It shows that the number of clusters in EELBCRP-2 is much fewer than LEACH.

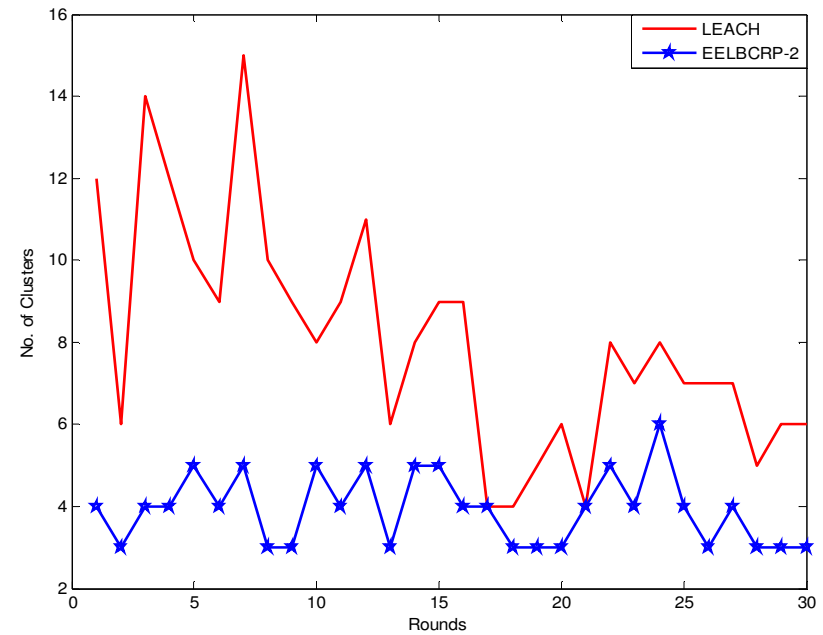

Figure 6. Number of Clusters

\subsection{Life time of WSN}

The result between the number of nodes alive and the number of rounds is shown by Fig [7]. The result obtained by measuring of time until the first node dies to time until the last node dies for 410 rounds. The first dead node appeared in round 97 for EELBCRP-2, in 82 rounds for LEACH and the last dead node appeared in 407 rounds for EELBCRP-2 and in 335 rounds for LEACH. It is observed that the EELBCRP-2 much better improves the life time of network than the LEACH protocol. 


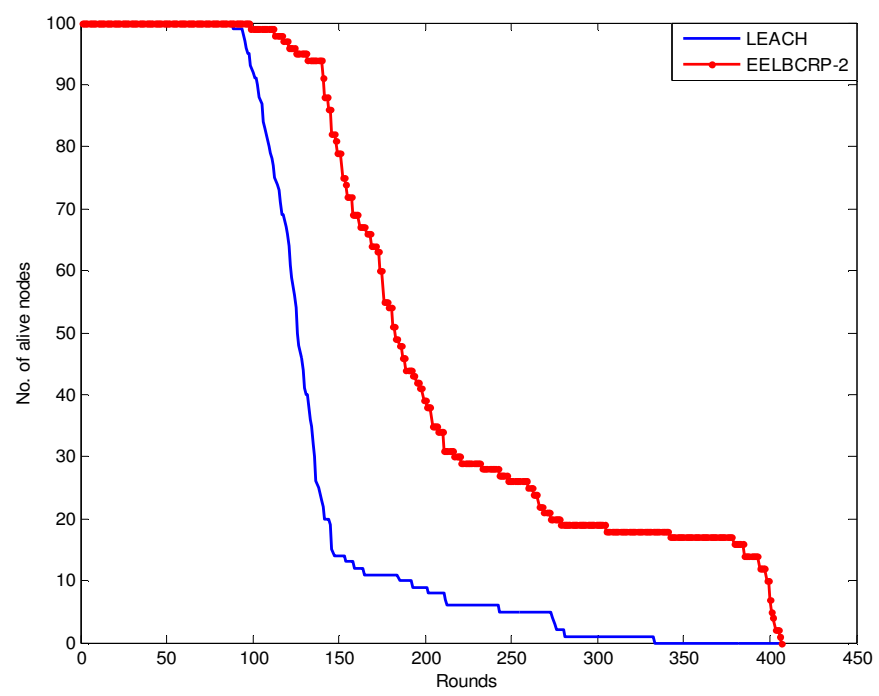

Figure 7. Network lifetime

\section{CONCLUSION AND FUTURE WORK}

In this paper, a level based clustering routing protocol has been proposed. The network model based on power levels is being developed. The mathematical formulae for choosing the cluster head are provided. The model developed is simulated in MATLAB.The simulation results of energy consumption of cluster heads, numbers of clusters and network lifetime are provided. It has been observed that the energy consumed by CHs for each round in EELBCRP-2 is much lower than that in LEACH. For example, after the 20 rounds, the LEACH consumed the about $42 \%$ of the initial energy while in EELBCRP is about $15 \%$. It has been also observed that the number of clusters in EELBCRP-2 is fewer than LEACH. Finally, it is concluded that the performance of EELBCRP is better than LEACH.

In future research, we will study to optimize the number of levels to efficiently consume the energy of all nodes and improve the network lifetime. We also want to extend our algorithm to heterogeneous WSNs.

\section{ACKNOWLEDGEMENT}

This work was supported by council of scientific and industrial research, India for promotion of research and scientific excellence program. 
International Journal Of Advanced Smart Sensor Network Systems ( IJASSN ), Vol 2, No.2, April 2012

\section{REFERENCES}

[1] Holger Karl and Andreas Willig. "Protocols and Architecture for Wireless sensor networks," Wiley, 2005.ISBN:0470095105.

[2] Kemal Akkaya , Mohamed Younis, "A survey on routing protocols for wireless sensor networks," 2003 Elsevier B.V.

[3] Wendi RabinerHeinzelman, AnanthaChandrakasan, and HariBalakrishnan, "Energy-Efficient Communication Protocol for Wireless Microsensor Networks", Proceedings of the 33rd Hawaii International Conference on System Sciences - 2000

[4] Heinzelman W, Candrakasan A, Balakrisnan H. "AN Application-Specific Protocol Architecture for Wireless Microsensor Networks [J]”, IEEE Transaction on Wireless Networking, 2000, 1(4): 660670.

[5] SHANG Fengjun, "A Distributed Clustering Algorithm for Wireless Sensor Networks," Wuhan University Journal of Natural Sciences 2008, Vol.13 No.4, 385-390

[6] I.F. Akyildiz, W. Su, Y. Sankarasubramaniam, E. Cayirci, "Wireless sensor networks: a survey".

[7] Dr.Garimella Rama murthy, VasanthIyer, V.BhawaniRadhika, "Level Controlled Clustering In Wireless Sensor Networks," 2008 IEEE.

[8] Andrea Goldsmith, "Wireless Communications," Cambridge University Press, 2005, pp. 28-29

[9] F. Akyildiz, W. Su, Y. Sankarasubramaniam, and E. Cayirci,“A Survey on Sensor Networks,” IEEE CommunicationsMagazine, Aug. 2002.

[10] M. Tubaishat, S. Madria, “Sensor Networks: An Overview”,IEEE Potentials, Volume 22, Issue 2, pages 20 -23, April2003.

[11] Huang Lu, Jie Li, Guojun Wang, "A Novel Energy Efficient Routing Algorithm forHierarchicallyClusteredWireless Sensor Networks," 2009 IEEE.

[12] Wen-Wen Huang, Ya-Li Peng ,Jian Wen, Min Yu, "Energy-Efficient Multi-hop Hierarchical Routing Protocol for Wireless Sensor Networks," 2009 IEEE.

[13] Meenakshi Diwakar, Sushil Kumar," Energy Efficient Hierarchical Clustering Routing Protocol For Wireless Sensor Networks", CCSIT 2012, Part I, LNICST 84, pp.409-420, Springer.

\section{BIOGRAPHY}

Meenakshi Diwakar received her M. Tech degrees in Computer Science from School of Computer and Systems Sciences, Jawaharlal Nehru University, New Delhi, India in 2009, M.Sc. and B.Sc.in Mathematics from M.J.P. Rohilkhand University, Bareilly, India in 2003 and 2001 respectively. She is currently pursuing Ph.D (Computer Science) from School of Computer and Systems Sciences, Jawaharlal Nehru University, New Delhi, India. Her current research interest includes Wireless Sensor Networks.

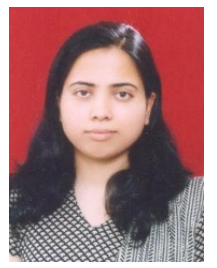

Sushil Kumar received his MCA and M. Tech degrees in Computer Science from School of Computer and Systems Sciences, Jawaharlal Nehru University, New Delhi, India in 1997 and 1999, respectively, and B.S. degree in Mathematics from Kanpur University, India in 1993. He is currently working as Assistant Professor at School of Computer and Systems Sciences, Jawaharlal Nehru University, New Delhi, India. He is pursuing Ph.D (Computer Science) from School of Computer and Systems Sciences, Jawaharlal Nehru University, New Delhi, India. His current research interest includes

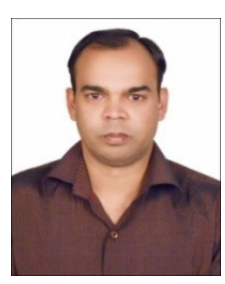
Mobile Ad hoc Networks, and Wireless Sensor Networks. 